Pacific

Journal of

Mathematics

AN INVARIANT SUPERTRACE FOR THE CATEGORY OF REPRESENTATIONS OF LIE SUPERALGEBRAS OF TYPE I

NATHAN GeER AND Bertrand Patureau-Mirand 


\title{
AN INVARIANT SUPERTRACE FOR THE CATEGORY OF REPRESENTATIONS OF LIE SUPERALGEBRAS OF TYPE I
}

\author{
NAthan GeEr And Bertrand Patureau-Mirand
}

\begin{abstract}
In this paper we give a renormalization of the supertrace on the category of representations of Lie superalgebras of type I, by a kind of modified superdimension. The genuine superdimensions and supertraces are generically zero. However, these modified superdimensions are nonzero and lead to a kind of supertrace which is nontrivial and invariant. As an application we show that this new supertrace gives rise to a nonzero bilinear form on a space of invariant tensors of a Lie superalgebra of type I. The results of this paper are completely classical results in the theory of Lie superalgebras but surprisingly we cannot prove them without using quantum algebra and low-dimensional topology.
\end{abstract}

\section{Introduction}

The theory of quantum groups and classical representation theory of Lie algebras has been widely and productively used in low-dimensional topology. There are few examples of low-dimensional topology or quantum groups being used to produce results in the classical theory of Lie algebras. Good examples of such work include the theory of crystal bases [Kashiwara 1990] and the use of the Kontsevich integral to give a new proof of the multiplicativity of the Duflo-Kirillov map $S(\mathfrak{g}) \rightarrow U(\mathfrak{g})$ for metrized Lie (super-)algebras $\mathfrak{g}$ [Bar-Natan et al. 2003]. In this paper we use low-dimensional topology and quantum groups to define a nontrivial kind of supertrace on the category of representations of a Lie superalgebra of type I. The genuine supertrace is generically zero on such a category Proposition 2.2.

In [Geer and Patureau-Mirand 2006; Geer et al. 2007], we give a renormalization of the Reshetikhin-Turaev quantum invariants, by modified quantum dimensions. In the case of simple Lie algebras these modified quantum dimensions are proportional to the genuine quantum dimensions. For Lie superalgebras of type I the genuine quantum dimensions are generically zero but the modified quantum dimensions are nonzero and lead to nontrivial link invariants. In this case the

MSC2000: primary 17B99; secondary 17B37.

Keywords: Lie superalgebra, tensor category, quantum group, knot, trace.

Geer's work was partially supported by NSF grant DMS-0706725. 
modified quantum dimension of a quantized module is given by an explicit formula which is determined by the underlying Lie superalgebra module. In this paper we take the classical limit of the modified quantum dimension to obtain a modified superdimension. Then we use this modified superdimension to renormalize the supertrace and define a nontrivial bilinear form on a space of invariant tensor.

Our proof that the modified supertrace is well defined and has the desired properties is as follows. We first formulate the desired statements at the level of the Lie superalgebra. Then we "deform" these statements to the quantum level and use low-dimensional topology to prove these "deformed" statements. Taking the classical limit we recover the original statements. To make this proof precise we use the Etingof-Kazhdan theory of quantization.

\section{Preliminaries}

1.1. The category $\mathrm{SV}$ of superspaces. A superspace is a $\mathbb{Z}_{2}$-graded vector space $V=V_{\overline{0}} \oplus V_{\overline{1}}$ over $\mathbb{C}$. We denote the parity of an homogeneous element $x \in V$ by $\bar{x} \in \mathbb{Z}_{2}$. We say $x$ is even if it lies in $V_{\overline{0}}$, and odd if it lies in $V_{\overline{1}}$ ). We now recall some basic features and conventions concerning the category of superspaces.

The theory of superspaces follows the rule "whenever you permute two odd elements in an expression, put a - sign". With this in mind, many concepts of linear algebra have super analogs. These analogs have new and different properties which are relevant to this paper. Let us discuss some of these differences.

In all the following, elements of superspaces are generally assumed to be homogeneous and thus their parity is well defined. The definitions must be generalized by linearity for nonhomogeneous elements.

The category SV of superspaces is a category whose objects are superspaces. The morphisms in SV between two objects $U$ and $V$ denoted by $\operatorname{Hom}_{\mathbb{C}}(U, V)$ is the superspace of linear maps with the parity given by

$$
\begin{aligned}
& \operatorname{Hom}_{\mathbb{C}}(U, V)_{\overline{0}}=\operatorname{Hom}_{\mathbb{C}}\left(U_{\overline{0}}, V_{\overline{0}}\right) \oplus \operatorname{Hom}_{\mathbb{C}}\left(U_{\overline{1}}, V_{\overline{1}}\right), \\
& \operatorname{Hom}_{\mathbb{C}}(U, V)_{\overline{1}}=\operatorname{Hom}_{\mathbb{C}}\left(U_{\overline{0}}, V_{\overline{1}}\right) \oplus \operatorname{Hom}_{\mathbb{C}}\left(U_{\overline{1}}, V_{\overline{0}}\right) .
\end{aligned}
$$

This category is "supermonoidal" with the super version of the operator $\otimes$ (we denote by $\underline{\otimes}$ the usual tensor product in the category Vect):

For two objects $U, V$ of SV their tensor product is the vector space $U \otimes V$ with the $\mathbb{Z}_{2}$-grading given by

$$
\begin{aligned}
& (U \otimes V)_{\overline{0}}=U_{\overline{0}} \underline{\otimes} V_{\overline{0}} \oplus U_{\overline{1}} \underline{\otimes} V_{\overline{1}}, \\
& (U \otimes V)_{\overline{1}}=U_{\overline{0}} \underline{\otimes} V_{\overline{1}} \oplus U_{\overline{1}} \underline{\otimes} V_{\overline{0}} .
\end{aligned}
$$

and for morphisms $f \in \operatorname{Hom}_{\mathbb{C}}\left(U, U^{\prime}\right)$ and $g \in \operatorname{Hom}_{\mathbb{C}}\left(V, V^{\prime}\right), f \otimes g$ is given by 


$$
f \otimes g=\left\{\begin{array}{r}
f \underline{\otimes} g \text { on } U_{\overline{0}} \underline{\otimes} V, \\
(-1)^{\bar{g}} f \underline{\otimes} g \text { on } U_{\overline{1}} \underline{\otimes} V .
\end{array}\right.
$$

Thus $(f \otimes g)(x \otimes y)=(-1)^{\bar{g} . \bar{x}} f(x) \otimes g(y)$. When $U$ and $V$ are finite-dimensional, this tensor product realizes an isomorphism:

$$
\operatorname{Hom}_{\mathbb{C}}\left(U, U^{\prime}\right) \otimes \operatorname{Hom}_{\mathbb{C}}\left(V, V^{\prime}\right) \simeq \operatorname{Hom}_{\mathbb{C}}\left(U \otimes V, U^{\prime} \otimes V^{\prime}\right) .
$$

Let $\mathrm{SV}_{\overline{0}}$ be the subcategory of $\mathrm{SV}$ with the same objects but only even morphisms (i.e., $\left.\operatorname{Hom}_{\mathrm{SV}_{\overline{0}}}(U, V)=\operatorname{Hom}_{\mathbb{C}}(U, V)_{\overline{0}}\right)$. The tensor product $\otimes$ restricted to $\mathrm{SV}_{\overline{0}}$ is the usual bifunctor of Vect with an appropriate grading on objects. Moreover, $\mathrm{SV}_{\overline{0}}$ is a symmetric monoidal category with symmetry isomorphisms $\tau_{U, V}: U \otimes V \simeq V \otimes U$ given by the superpermutation $\tau_{U, V}(u \otimes v)=(-1)^{\bar{u} \cdot \bar{v}} v \otimes u$. The category $\mathrm{SV}$ is not a symmetric monoidal category because in general there are morphisms $f$ and $g$ with the property that $(\operatorname{Id} \otimes g) \circ(f \otimes I d) \neq(f \otimes I d) \circ(\operatorname{Id} \otimes g)$.

For a superspace $U$, the superdual $U^{*}$ is defined as the superspace $\operatorname{Hom}_{\mathbb{C}}(U, \mathbb{C})$. The tensor product gives the canonical isomorphism

$$
U^{*} \otimes V^{*}=\operatorname{Hom}_{\mathbb{C}}(U, \mathbb{C}) \otimes \operatorname{Hom}_{\mathbb{C}}(V, \mathbb{C}) \simeq \operatorname{Hom}_{\mathbb{C}}(U \otimes V, \mathbb{C} \otimes \mathbb{C})=(U \otimes V)^{*} .
$$

If $f \in \operatorname{Hom}_{\mathbb{C}}(U, V)$, the supertranspose of $f$ is the linear map $f^{*} \in \operatorname{Hom}_{\mathbb{C}}\left(V^{*}, U^{*}\right)$ given by

$$
f^{*}(\phi)=(-1)^{\bar{f} \cdot \bar{\phi}} \phi \circ f
$$

for $\phi \in V^{*}$. Then, if $f, g$ are composable morphisms of SV, we have

$$
(f \circ g)^{*}=(-1)^{\bar{f} \cdot \bar{g}} g^{*} \circ f^{*} .
$$

By convention the dual is a left dual:

- (left duality) $\operatorname{ev}_{V} \in \operatorname{Hom}_{\mathbb{C}}\left(V^{*} \otimes V, \mathbb{C}\right)$ is simply the contraction $\langle\phi, x\rangle=\phi(x)$.

- (right duality) $\mathrm{ev}_{V}^{\prime} \in \operatorname{Hom}_{\mathbb{C}}\left(V \otimes V^{*}, \mathbb{C}\right)$ is given by $\langle x, \phi\rangle=(-1)^{\bar{x} . \bar{\phi}} \phi(x)$

This defines a canonical isomorphism $V \rightarrow V^{* *}$ when $V$ is finite dimensional. Again here, when restricted to $\mathrm{SV}_{\overline{0}}$ the * became a functor, namely, the usual contravariant duality functor with some grading information.

The category $\mathfrak{g}$-Mod of $\mathfrak{g}$-modules. A Lie superalgebra is a superspace $\mathfrak{g}=\mathfrak{g}_{\overline{0}} \oplus \mathfrak{g}_{\overline{1}}$ with a superbracket $[]:, \mathfrak{g}^{\otimes 2} \rightarrow \mathfrak{g}$ that preserves the $\mathbb{Z}_{2}$-grading, is superantisymmetric $\left([x, y]=-(-1)^{\bar{x}} \bar{y}[y, x]\right)$, and satisfies the super-Jacobi identity [Kac 1977]. In this section, we assume that $\mathfrak{g}$ is a classical Lie superalgebra which means that the Lie algebra $\mathfrak{g}_{0}$ is reductive.

The universal enveloping algebra $U(\mathfrak{g})$ of $\mathfrak{g}$ is a Hopf superalgebra, that is, $U(\mathfrak{g})$ is a Hopf algebra object in $\mathrm{SV}_{\overline{0}}$. Let $\mathfrak{g}$-Mod be the category where objects are finite-dimensional superspaces with a structure of regular $\mathfrak{g}$-modules (that is, regular $U(\mathfrak{g})$-modules). By regular we mean that elements of the center of the 
reductive Lie algebra $\mathfrak{g}_{\overline{0}}$ act as diagonalisable endomorphisms of a $\mathfrak{g}$-module. It is equivalent to require that $V$ is semisimple as a $\mathfrak{g}_{0}$-module (i.e., $V$ splits as a direct sum of irreducible $\mathfrak{g}_{0}$-modules). The morphisms of $\mathfrak{g}$-Mod are the morphisms $f$ of SV that are ("super") $\mathfrak{g}$-linear:

$$
f(x . v)=(-1)^{\bar{x} \cdot \bar{f}} x . f(v) \text { for all } x \in \mathfrak{g} \text { and } v \in V .
$$

If $U$ and $V$ are two $\mathfrak{g}$-modules we denote by $\operatorname{Hom}_{\mathfrak{g}}(U, V)$ the superspace of $\mathfrak{g}$ module morphisms. The superspace $\operatorname{Hom}_{\mathfrak{g}}(U, V)$ should not be confused with $\operatorname{Hom}_{\mathbb{C}}(U, V)$ (where $U$ and $V$ are viewed as superspaces) which is naturally equipped with a $\mathfrak{g}$-module structure.

The structure of Hopf superalgebra on $U(\mathfrak{g})$ gives the tensor product of two $\mathfrak{g}$ modules a natural structure of $\mathfrak{g}$-modules and the tensor product of two $\mathfrak{g}$-linear morphisms is $\mathfrak{g}$-linear. Similarly, if $V$ is an object of $\mathfrak{g}$-Mod then the superspace $V^{*}$ is a $\mathfrak{g}$-module whose action is induced from the antipodal map of $U(\mathfrak{g})$. $\operatorname{Hom}_{\mathfrak{g}}(U, V)$ is canonically isomorphic to the superspace of invariant elements of $V \otimes U^{*}$ and so

$$
\operatorname{Hom}_{\mathfrak{g}}(U, V) \cong \operatorname{Hom}_{\mathfrak{g}}\left(\mathbb{C}, V \otimes U^{*}\right) .
$$

Let $\mathfrak{g}-\operatorname{Mod}_{\overline{0}}$ be the category whose objects are the objects of $\mathfrak{g}$-Mod and whose morphisms are morphisms of $\mathrm{SV}_{\overline{0}}$ which are $\mathfrak{g}$-linear. Then as above $\mathfrak{g}-\mathrm{Mod}_{\overline{0}}$ becomes a symmetric monoidal category with duality. Note that in general $\mathfrak{g}$-Mod is not such a category. This is the reason we require that the morphisms $\alpha$ and $\beta$ in the definition of $\mathscr{I}$ Proposition 1.2 are in $\mathfrak{g}-\operatorname{Mod}_{\overline{0}}$. In other words, the proof of Theorem 1 requires that we work in the category $\mathfrak{g}-\operatorname{Mod}_{\overline{0}}$.

1.2. Lie superalgebras of type I. In this subsection we recall notations and properties related to Lie superalgebras of type I.

Throughout the rest of the paper, let $\mathfrak{g}=\mathfrak{g}_{\overline{0}} \oplus \mathfrak{g}_{\overline{1}}$ be a Lie superalgebra of type $\mathrm{I}$, so $\mathfrak{g}$ is equal to $\mathfrak{s l}(m \mid n)$ or $\mathfrak{o s p}(2 \mid 2 n)$. We will assume that $m \neq n$. Let $\mathfrak{b}$ be the distinguished Borel subsuperalgebra of $\mathfrak{g}$. Then $\mathfrak{b}$ can be written as the direct sum of a Cartan subsuperalgebra $\mathfrak{h}$ and a positive nilpotent subsuperalgebra $\mathfrak{n}_{+}$. Moreover, $\mathfrak{g}$ admits a decomposition $\mathfrak{g}=\mathfrak{n}_{-} \oplus \mathfrak{h} \oplus \mathfrak{n}_{+}$. Let $W$ be the Weyl group of the even part $\mathfrak{g}_{0}$ of $\mathfrak{g}$.

Let $\Delta_{\overline{0}}^{+}$be the even positive roots and $\Delta_{\overline{1}}^{+}$) the odd ones. Let $\rho_{\overline{0}}$ and $\rho_{\overline{1}}$ denote the half-sum of all even and odd positive roots, respectively. Set $\rho=\rho_{\overline{0}}-\rho_{\overline{1}}$. A positive root is called simple if it cannot be decomposed into a sum of two positive roots.

A Cartan matrix associated to a Lie superalgebra is a pair consisting of a $r \times r$ matrix $A=\left(a_{i j}\right)$ and a set $\tau \subset\{1, \ldots, r\}$ determining the parity of the generators. 
Let $(A, \tau)$ be the Cartan matrix arising from $\mathfrak{g}$ and the distinguished Borel subsuperalgebra $\mathfrak{b}$. Here the set $\tau=\{s\}$ consists of only one element because of our choice of Borel subalgebra $\mathfrak{b}$.

By Proposition 1.5 of [Kac 1978] there exists $e_{i} \in \mathfrak{n}_{+}, f_{i} \in \mathfrak{n}_{-}$and $h_{i} \in \mathfrak{h}$ for $i=1, \ldots, r$ such that the Lie superalgebra $\mathfrak{g}$ is generated by $e_{i}, f_{i}, h_{i}$ where

$$
\left[e_{i}, f_{j}\right]=\delta_{i j} h_{i}, \quad\left[h_{i}, h_{j}\right]=0, \quad\left[h_{i}, e_{j}\right]=a_{i j} e_{j}, \quad\left[h_{i}, f_{j}\right]=-a_{i j} f_{j} .
$$

Note that these generators also satisfy the Serre relations and higher order Serre type relations [Yamane 1994].

There exist $d_{1}, \ldots, d_{r}$ in $\{ \pm 1, \pm 2\}$ such that the matrix $\left(d_{i} a_{i j}\right)$ is symmetric. Let $\langle.,$.$\rangle be the symmetric nondegenerate form on \mathfrak{h}$ determined by $\left\langle h_{i}, h_{j}\right\rangle=d_{j}^{-1} a_{i j}$. This form gives an identification of $\mathfrak{h}$ and $\mathfrak{h}^{*}$. Moreover, the form $\langle.,$.$\rangle induces a$ $W$-invariant bilinear form on $\mathfrak{h}^{*}$, which we will also denote by $\langle.,$.$\rangle .$

1.3. Irreducible $\mathfrak{g}$-modules. Modules over Lie superalgebras of type I are different in nature than modules over semisimple Lie algebras. For example, each Lie superalgebra of type I has one parameter families of irreducible modules. Any module in such a family has superdimension zero and so the supertrace of an endomorphism of such a module is zero - see Equality (3).

There is a super analog of the Schur's Lemma [Kac 1978]:

Lemma 1.1 (Schur's Lemma). Let $V$ be a superspace, $M$ an irreducible family of operators from $\operatorname{End}_{\mathrm{SV}}(V)$, and $C(\mathcal{M})=\left\{a \in \operatorname{End}_{\mathrm{SV}}(V):[a, m]=0, \forall m \in \mathcal{M}\right.$. Then either

- $C(M)$ is generated by $\operatorname{Id}_{V}$, or

- $C(M)$ is generated by $\operatorname{Id}_{V}$ and $s$ where $s$ is an odd endomorphism of $V$ such that $s^{2}=\operatorname{Id}_{V}$ (and in particular $\left.\operatorname{dim} V_{\overline{0}}=\operatorname{dim} V_{\overline{1}}\right)$.

For $\mathfrak{g}$-Mod the situation is simplified: for any $\mathfrak{g}$-module $V$ of $\mathfrak{g}$-Mod,

$$
V \text { is irreducible } \Rightarrow \operatorname{End}_{\mathfrak{g}}(V)=\mathbb{C} \operatorname{Id}_{V} \text {. }
$$

This follows from the fact that any module of $\mathfrak{g}$-Mod is regular and thus it is a weight module (a direct sum of its weight spaces) and any irreducible module of $\mathfrak{g}$-Mod is a highest weight module with unique (up to a scalar) highest weight vector. Kac [1977] gives a construction of these irreducible modules: Let $\lambda \in \mathfrak{h}^{*}$ be a linear functional on $\mathfrak{h}$. Kac defined an irreducible highest weight $\mathfrak{g}$-module $V(\lambda)$ of weight $\lambda$ with a highest weight vector $v_{0}$ having the property that $h . v_{0}=\lambda(h) v_{0}$ for all $h \in \mathfrak{h}$ and $\mathfrak{n}_{+} v_{0}=0$. Let $a_{i}=\lambda\left(h_{i}\right)$. Kac showed that $V(\lambda)$ is finitedimensional if and only if $a_{i} \in \mathbb{N}$ for $i \neq s$. Therefore, $a_{s}$ can be an arbitrary complex number. Irreducible finite-dimensional $\mathfrak{g}$-modules are divided into two classes: typical and atypical. 
There are many equivalent definitions for a weight module to be typical [Kac 1978]. Here we say that $V(\lambda)$ is typical if it splits in any finite-dimensional regular $\mathfrak{g}$-module (i.e., if it is a submodule or a factor-module of a finite-dimensional regular $\mathfrak{g}$-module then it is a direct summand). By Theorem 1 of [Kac 1978] this is equivalent to requiring that

$$
\langle\lambda+\rho, \alpha\rangle \neq 0
$$

for all $\alpha \in \Delta_{\overline{1}}^{+}$. If $V(\lambda)$ is (a)typical we will say the weight $\lambda$ is (a)typical.

In Section 2 we construct a trace on the "ideal" generated by typical modules. With this in mind let us recall some properties of these modules. The space of typical weights is dense in the space of weights corresponding to finite-dimensional modules. In particular, if $a_{i} \in \mathbb{N}$ for $1 \leq i \leq r$ and $i \neq s$ then there are only finitely many atypical weights with $a_{i}=\lambda\left(h_{i}\right)$. Furthermore, if $\lambda$ is atypical then $a_{s}=\lambda\left(h_{s}\right) \in \mathbb{Z}$. Thus, the name typical is fitting.

For any object $V$ of $\mathfrak{g}$-Mod whose $\mathbb{Z}_{2}$ grading is given by $V=V_{\overline{0}} \oplus V_{\overline{1}}$ let $\operatorname{sdim}(V)=\operatorname{dim}\left(V_{\overline{0}}\right)-\operatorname{dim}\left(V_{\overline{1}}\right)$ be the superdimension of $V$. By Proposition 2.10 of [Kac 1978], if $V$ is a typical $\mathfrak{g}$-module then $\operatorname{sdim}(V)=0$. This vanishing can make other mathematical objects trivial. For example, the supertrace on endomorphisms of a typical module Proposition 2.2 and quantum invariants of links arising from Lie superalgebras [Geer and Patureau-Mirand 2006].

Fix a typical module $V_{0}$. Let $\Phi_{V_{0}}$ be the set of objects $V$ of $\mathfrak{g}$-Mod such that there exists an object $W$ of $\mathfrak{g}$-Mod and even $\mathfrak{g}$-linear morphisms $\alpha: V \rightarrow V_{0} \otimes W$ and $\beta: V_{0} \otimes W \rightarrow V$ with $\beta \circ \alpha=\mathrm{Id}_{V}$.

Proposition 1.2. The definition of $\Phi_{V_{0}}$ does not depend on the choice of $V_{0}$, i.e., $\Phi_{V_{0}}=\mathscr{I}_{V_{1}}$ for any two typical modules $V_{0}$ and $V_{1}$.

The set $\mathscr{I}_{V_{0}}$ is an ideal in the sense that for any $V, V^{\prime} \in \mathscr{I}_{V_{0}}$ and $W \in \mathfrak{g}$-Mod we have $V \otimes W \in \mathscr{I}_{V_{0}}$ and $V \oplus V^{\prime} \in \mathscr{I}_{V_{0}}$.

We define $\mathscr{I}$ to be the set $\mathscr{I}_{V}$ where $V$ is any typical module, which is well defined by the proposition.

Proof. We will prove the first statement; the second follows easily from the definition of $\mathscr{I}_{V_{0}}$. First, $W \in \mathscr{I}_{V}$ if and only if $\mathscr{I}_{W} \subset \mathscr{I}_{V}$. We will use this fact in the remainder of the proof.

As mentioned above irreducible finite-dimensional $\mathfrak{g}$-modules are in one to one correspondence with $\mathbb{N}^{r-1} \times \mathbb{C}$. We will denote $V_{\alpha}^{\bar{c}}$ as the module corresponding to $(\bar{c}, \alpha) \in \mathbb{N}^{r-1} \times \mathbb{C}$. Let $V_{\alpha}^{\overline{0}}$ and $V_{\beta}^{\bar{c}}$ be typical modules. From the character formula for typical modules we know that $V_{\beta}^{\bar{c}}$ is a submodule of $V_{\alpha}^{\overline{0}} \otimes V_{\beta-\alpha}^{\bar{c}}$. Since typical modules always split we have $V_{\beta}^{\bar{c}} \in \Phi_{V_{\alpha}^{\overline{0}}}$ and so $\mathscr{I}_{V_{\bar{\beta}}^{\bar{c}}} \subset \Phi_{V_{\alpha}^{\overline{0}}}$. 
On the other hand, from the discussion in the previous paragraph we have $\operatorname{Hom}_{\mathfrak{g}}\left(V_{\alpha}^{\overline{0}} \otimes V_{\beta-\alpha}^{\bar{c}}, V_{\beta}^{\bar{c}}\right) \neq 0$, implying $\operatorname{Hom}_{\mathfrak{g}}\left(V_{\alpha}^{\overline{0}}, V_{\beta}^{\bar{c}} \otimes\left(V_{\beta-\alpha}^{\bar{c}}\right)^{*}\right) \neq 0$. Therefore, as $V_{\alpha}^{\overline{0}}$ is typical, $V_{\alpha}^{\overline{0}} \in \mathscr{I}_{V_{\beta}^{\bar{c}}}$ and so $\mathscr{I}_{V_{\alpha}^{\overline{0}}} \subset \mathscr{I}_{V_{\bar{\beta}}^{\bar{c}}}$.

\section{A trace}

In this section we define a nonzero supertrace on $\operatorname{End}_{\mathfrak{g}}(V)$ for $V \in \mathscr{I}$. First, let us prove that the usual supertrace on $\operatorname{End}_{\mathfrak{g}}(V)$ is zero.

Let $V$ be a superspace and let $\left\{v_{i}\right\}$ be a basis of $V$ with homogeneous vectors. Let $\left\{v_{i}^{*}\right\}$ be the dual basis of $V^{*}$. We have that $\bar{v}_{i}^{*}=\bar{v}_{i}=\bar{v}_{i} \cdot \bar{v}_{i}^{*}$. Define the supertrace on $\operatorname{End}_{\mathbb{C}}(V)$ to be the function $\operatorname{str}_{V}: \operatorname{End}_{\mathbb{C}}(V) \rightarrow \mathbb{C}$ given by $f \mapsto$ $\sum_{i}(-1)^{\bar{v}_{i}} v_{i}^{*}\left(f\left(v_{i}\right)\right)$. Then str has the property that if $f \in \operatorname{Hom}_{\mathbb{C}}(V, W)$ and $g \in$ $\operatorname{Hom}_{\mathbb{C}}(W, V)$ then $\operatorname{str}_{W}(f \circ g)=(-1)^{\bar{f} \cdot \bar{g}} \operatorname{str}_{V}(g \circ f)$.

Let us define the partial supertrace that is a generalization of the supertrace. For this, we first define the evaluation and coevaluation morphisms ev $v_{V}: V \otimes V^{*} \rightarrow \mathbb{C}$ and $\operatorname{coev}_{V}: \mathbb{C} \rightarrow V \otimes V^{*}$ given by $v \otimes f \mapsto(-1)^{\bar{f} \bar{v}} f(v)$ and $1 \mapsto \sum_{i} v_{i} \otimes v_{i}^{*}$, respectively.

Definition 2.1. Let $U$ and $V$ be superspaces and $f \in \operatorname{End}_{\mathbb{C}}(U \otimes V)$. Then we call the partial supertrace of $f$ the endomorphism

$$
\operatorname{ptr}(f)=\left(\operatorname{Id}_{U} \otimes \operatorname{ev}_{V}\right) \circ\left(f \otimes \operatorname{Id}_{V^{*}}\right) \circ\left(\operatorname{Id}_{U} \otimes \operatorname{coev}_{V}\right) \in \operatorname{End}_{\mathbb{C}}(U) .
$$

For $f$ as in Definition 2.1 we have $\operatorname{str}_{U \otimes V}(f)=\operatorname{str}_{U}(\operatorname{ptr}(f))$. In addition, if $f \in \operatorname{End}_{\mathfrak{g}}(U \otimes V)$ then $\operatorname{ptr}(f) \in \operatorname{End}_{\mathfrak{g}}(U)$.

Let $V$ be an element of $\mathscr{I}=\mathscr{I}_{V_{0}}$ and $f \in \operatorname{End}_{\mathfrak{g}}(V)$. Choose morphisms $\alpha$ : $V_{0} \otimes W \rightarrow V$ and $\beta: V \rightarrow V_{0} \otimes W$ such that $\alpha \circ \beta=\operatorname{Id}_{V}$. Then $\operatorname{ptr}(\beta \circ f \circ \alpha)$ is an invariant map of $V_{0}$ and $\operatorname{sop} \operatorname{ptr}(\beta \circ f \circ \alpha)=\mathfrak{c} \operatorname{Id}_{V_{0}}$ for some $\mathfrak{c} \in \mathbb{C}$. We define the bracket of the triple $(f, \alpha, \beta)$ to be $\langle f ; \alpha ; \beta\rangle=\mathfrak{c}$.

Proposition 2.2. Let $V \in \mathscr{I}$ and $f \in \operatorname{End}_{\mathfrak{g}}(V)$ then $\operatorname{str}_{V}(f)=0$.

Proof. Using the notation above, we have

$$
\operatorname{str}_{V}(f)=\operatorname{str}_{V}(f \circ \alpha \circ \beta)=\operatorname{str}_{V_{0} \otimes W}(\beta \circ f \circ \alpha)=\operatorname{str}_{V_{0}}(\operatorname{ptr}(\beta \circ f \circ \alpha)) .
$$

But $\operatorname{ptr}(\beta \circ f \circ \alpha)=\langle f ; \alpha ; \beta\rangle \operatorname{Id}_{V_{0}}$ so

$$
\operatorname{str}_{V}(f)=\operatorname{str}_{V_{0}}\left(\langle f ; \alpha ; \beta\rangle \operatorname{Id}_{V_{0}}\right)=\langle f ; \alpha ; \beta\rangle \operatorname{sdim}\left(V_{0}\right)=0
$$

as the superdimension of $V_{0}$ is zero.

Definition 2.3. Let $\mathrm{d}:\{$ typical modules $\} \rightarrow \mathbb{C}$ be the function defined by

$$
\mathrm{d}(V(\lambda))=\prod_{\alpha \in \Delta_{\overline{0}}^{+}} \frac{\langle\lambda+\rho, \alpha\rangle}{\langle\rho, \alpha\rangle} / \prod_{\alpha \in \Delta_{\overline{1}}^{+}}\langle\lambda+\rho, \alpha\rangle .
$$


Note that Equation (4) implies that $d$ is well defined. As an example, if $\mathfrak{g}=$ $\mathfrak{s l}(n \mid 1)$ with $n \geq 2$, and $\lambda=(0, \ldots, 0 \mid a)$ with $a \in \mathbb{C} \backslash\{0,-1, \ldots, 1-n\}$, we have $\mathrm{d}(V(\lambda))=\prod_{i=0}^{n-1} 1 /(a+i)$.

Theorem 1. Let $V \in \mathscr{I}$ and $f \in \operatorname{End}_{\mathfrak{g}}(V)$. Choose a typical module $V_{0}$, and morphisms $\alpha \in \operatorname{Hom}_{\mathfrak{g}}\left(V_{0} \otimes W, V\right)_{\overline{0}}$ and $\beta \in \operatorname{Hom}_{\mathfrak{g}}\left(V, V_{0} \otimes W\right)_{\overline{0}}$ such that $\alpha \circ \beta=$ $\mathrm{Id}_{V}$. Then

$$
\operatorname{str}_{V}^{\prime}(f)=\mathrm{d}\left(V_{0}\right)\langle f ; \alpha ; \beta\rangle
$$

depends only on $f$; it does not depend on the choice of $V_{0}, \alpha$ or $\beta$. Furthermore, str' $^{\prime}$ is a trace in the following sense: for any $V, V^{\prime} \in \Phi$ and any $\mathfrak{g}$-module $U$,

(a) $\operatorname{str}_{V}^{\prime}: \operatorname{End}_{\mathfrak{g}}(V) \rightarrow \mathbb{C}$ is linear.

(b) $\operatorname{str}_{V^{\prime}}^{\prime}(f \circ g)=(-1)^{\bar{g} \bar{f}} \operatorname{str}_{V}^{\prime}(g \circ f)$ for any $f \in \operatorname{Hom}_{\mathfrak{g}}\left(V, V^{\prime}\right), g \in \operatorname{Hom}_{\mathfrak{g}}\left(V^{\prime}, V\right)$.

(c) $\operatorname{str}_{V \otimes U}^{\prime}(f \otimes g)=\operatorname{str}_{V}^{\prime}(f) \operatorname{str}_{U}(g)$ for any $f \in \operatorname{End}_{\mathfrak{g}}(V)$ and any $g \in \operatorname{End}_{\mathfrak{g}}(U)$, in particular $\operatorname{str}^{\prime}(f \otimes g)=\operatorname{str}(g)=0$ if $U \in \mathscr{I}$.

(d) $\operatorname{str}_{V \otimes U}^{\prime}(f)=\operatorname{str}_{V}^{\prime}(\operatorname{ptr}(f))$ for any $f \in \operatorname{End}_{\mathfrak{g}}(V \otimes U)$.

The proof of Theorem 1 will be given in Section 4. Let us now make a few comments about this theorem. First, remark that property (d) implies property (c). Next, property (d) implies a kind of invariance for str'. Let us make this statement more precise.

Let $U, U^{\prime}$ be $\mathfrak{g}$-modules and $V, V^{\prime}$ be in $\mathscr{I}$. The following spaces of morphisms are canonically isomorphic:

$\operatorname{Hom}_{\mathfrak{g}}\left(\operatorname{Hom}_{\mathbb{C}}\left(U^{\prime}, V^{\prime}\right), \operatorname{Hom}_{\mathbb{C}}(U, V)\right) \cong \operatorname{Hom}_{\mathfrak{g}}\left(U \otimes V^{\prime}, V \otimes U^{\prime}\right)$

$$
\cong \operatorname{Hom}_{\mathfrak{g}}\left(V^{\prime} \otimes U, U^{\prime} \otimes V\right) \cong \operatorname{Hom}_{\mathfrak{g}}\left(\operatorname{Hom}_{\mathbb{C}}(V, U), \operatorname{Hom}_{\mathbb{C}}\left(V^{\prime}, U^{\prime}\right)\right) .
$$

Let $\Psi \in \operatorname{Hom}_{\mathfrak{g}}\left(\operatorname{Hom}_{\mathbb{C}}\left(U^{\prime}, V^{\prime}\right), \operatorname{Hom}_{\mathbb{C}}(U, V)\right)$ and let $h, h^{\#}, \Psi^{\#}$ be the corresponding morphisms in the other three spaces, respectively. We have $h^{\#}=\tau \circ h \circ \tau$ where $\tau$ is the superpermutation. Also, if $f \in \operatorname{Hom}_{\mathbb{C}}\left(U^{\prime}, V^{\prime}\right)$ and $g \in \operatorname{Hom}_{\mathbb{C}}(V, U)$ then $\Psi(f)=\operatorname{ptr}\left(h \circ\left(\operatorname{Id}_{U} \otimes f\right)\right)$ and $\Psi^{\#}(g)=\operatorname{ptr}\left(h^{\#} \circ\left(\operatorname{Id}_{V^{\prime}} \otimes g\right)\right)$ (here we use a generalization of the partial trace ptr: $\operatorname{Hom}(A \otimes C, B \otimes C) \rightarrow \operatorname{Hom}(A, B))$. Thus, applying property (d), we get that

$$
\operatorname{str}^{\prime}(\Psi(f) \circ g)=(-1)^{\bar{\Psi} \cdot \bar{f}} \operatorname{str}^{\prime}\left(f \circ \Psi^{\#}(g)\right) .
$$

Indeed,

$$
\begin{aligned}
\operatorname{str}^{\prime}(\Psi(f) \circ g) & =\operatorname{str}^{\prime}\left(\operatorname{ptr}\left(h \circ\left(\operatorname{Id}_{U} \otimes f\right)\right) \circ g\right)=(-1)^{\bar{g} \cdot \bar{f}} \operatorname{str}^{\prime}(\operatorname{ptr}(h \circ(g \otimes f))) \\
& =(-1)^{\bar{g} \cdot \bar{f}} \operatorname{str}^{\prime}(h \circ(g \otimes f))=\operatorname{str}^{\prime}\left(h^{\#} \circ(f \otimes g)\right) \\
& =\operatorname{str}^{\prime}\left(\operatorname{ptr}\left(h^{\#} \circ(f \otimes g)\right)\right)=(-1)^{\bar{g} \cdot \bar{f}} \operatorname{str}^{\prime}\left(\operatorname{ptr}\left(h^{\#} \circ\left(\operatorname{Id}_{V^{\prime}} \otimes g\right)\right) \circ f\right) \\
& =(-1)^{\bar{\Psi} \cdot \bar{f}} \operatorname{str}^{\prime}\left(f \circ \Psi^{\#}(g)\right) .
\end{aligned}
$$


The results of this section can be stated in the language of symmetric monoidal category with duality or more generally ribbon categories. We will not make this formalism precise, however we will end this section by giving the following graphs which we hope will shed light on the above results. For more details on ribbon categories see [Turaev 1994].

Here we will represent morphisms with ribbon graphs, which are read from bottom to top. The tensor product of two morphisms is represented by setting the two corresponding graphs next to each other. For example, if $f: V \rightarrow V^{\prime}$ and $g: U \rightarrow U^{\prime}$ are even morphism of $\mathfrak{g}$-Mod then we represent $f$ and $f \otimes g$ by

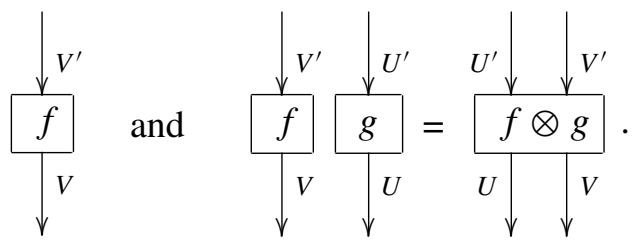

Let the graphs $V \smile$ and $V \circlearrowleft$ represent the morphisms ev $\operatorname{Ca}_{V}: V \otimes V^{*} \rightarrow \mathbb{C}$ and $\operatorname{coev}_{V}: \mathbb{C} \rightarrow V \otimes V^{*}$, respectively.

Let $g: V \rightarrow V$ be an even invariant morphism of a $\mathfrak{g}$-module $V$ and let $G$ be a ribbon graph representing $g$, as in Equation (6). If $V$ is simple then the morphism $g$ is a scalar times the identity, which we denote by $\langle g\rangle=\langle G\rangle$.

The elements $\operatorname{str}_{V}(g)$ and $\operatorname{str}_{V}^{\prime}(g)$ can be represented by

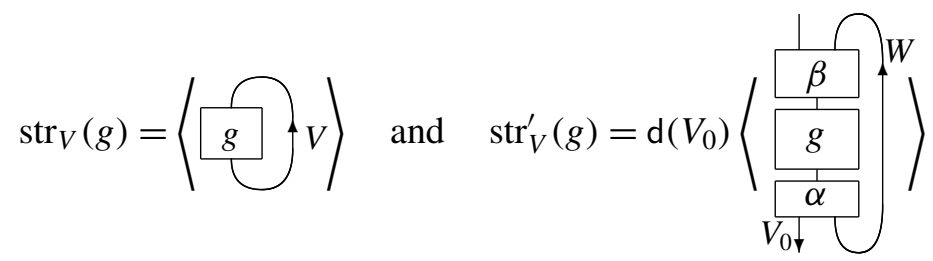

where we require $V \in \mathscr{I}$ in the second case. When $V$ is simple the supertrace can be rewritten as

$$
\operatorname{str}_{V}(g)=\left\langleV \bigcirc \left\langle\left\langle\frac{\downarrow^{V}}{\frac{g}{\downarrow_{V}}}\right\rangle=\operatorname{sdim}(V)\left\langle\frac{\downarrow^{V}}{\frac{g}{\downarrow_{V}}}\right\rangle\right.\right.
$$

where $\operatorname{sdim}(V)=0$ if $V$ is typical. Also, when $V$ is a typical module the $\operatorname{str}^{\prime}$ becomes

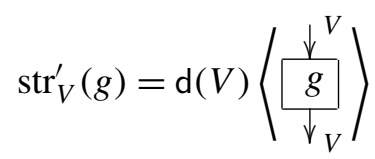

Thus, the function $d$ can be thought of as a nonzero replacement of the usual superdimension. Moreover, $d$ can be thought of as the classical analogue of the modified quantum dimensions defined in [Geer et al. 2007]. 
If $f: V \rightarrow V^{\prime}$ is an even invariant morphism let $f^{*}:\left(V^{\prime}\right)^{*} \rightarrow V^{*}$ be the "supertranspose" of $f$. We can represent $f^{*}$ by

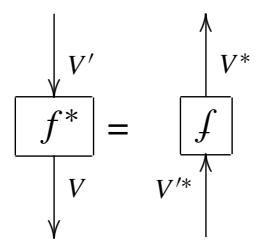

We will use the "supertranspose" in the next section.

\section{Invariant tensors}

In this section we define a nontrivial bilinear form on a space of invariant tensors of $\mathfrak{g}$. The standard bilinear form on $\mathfrak{g}$ is zero on this space of tensors.

Let $V$ be an object of $\mathfrak{g}$-Mod and let $T(V)=\oplus_{i} T(V)_{i}$ be the tensor algebra of $V$, where $T(V)_{i}$ is the space $V^{\otimes i}$. Let $T(V)^{\mathfrak{g}}$ be the invariant tensors of $T(V)$.

\section{Lemma 3.1. All invariant tensors of $T(\mathfrak{g})$ are even.}

Proof. We will prove the lemma for $\mathfrak{g}=\mathfrak{s l}(m \mid n)$, the prove for $\mathfrak{o s p}(2 \mid 2 n)$ is similar. We can identify $\mathfrak{s l}(m \mid n)$ with the Lie superalgebra of supertrace zero $(m+n) \times$ $(m+n)$ matrices. This standard representation is obtained by sending $e_{i}$ to the elementary matrix $E_{i, i+1}, f_{i}$ to $E_{i+1, i}, h_{i}$ to $E_{i, i}-E_{i+1, i+1}$ if $i \neq m$ and $h_{m}$ to $E_{m, m}+E_{m+1, m+1}$. The Cartan subalgebra $\mathfrak{h}$ with basis $\left\{h_{i}\right\}$ is contained in the space of diagonal matrices $X$. The space $X^{*}$ has a canonical basis $\left\{\epsilon_{1}, \ldots \epsilon_{m+n}\right\}$ which is dual to the basis formed by the matrices $\left\{E_{i, i}\right\}$. Set $\delta_{i}=\epsilon_{i+m}$, then $\mathfrak{h}$ is the kernel of the supertrace $\operatorname{str}=\sum \epsilon_{i}-\sum \delta_{j}$. Therefore, $\mathfrak{h}^{*}$ is the quotient of $X^{*}$ by the supertrace.

Let $\Lambda \subset \mathfrak{h}$ be the root lattice generated by the positive roots. Let $f: \Lambda \rightarrow \mathbb{Z}$ be the linear function determined by $\epsilon_{i} \mapsto n$ and $\delta_{j} \mapsto m$ (note that $\operatorname{str} \mapsto 0$ ). By definition the simple positive even roots $\epsilon_{i}-\epsilon_{j}$ and $\delta_{i}-\delta_{j}$ map to zero and the simple positive odd roots $\epsilon_{i}-\delta_{j}$ map to $-(m-n)$. Therefore, the image of $f$ is $(m-n) \mathbb{Z}$ and $f$ induces a linear map $\bar{f}: \Lambda \rightarrow \mathbb{Z} / 2 \mathbb{Z}$ given by $\alpha \mapsto \frac{f(\alpha)}{m-n}$ modulo 2. The map $\bar{f}$ in turn induces a map on the weight vectors of $T(\mathfrak{g})$ (which we also denote by $\bar{f})$ that satisfies $\bar{f}(x \otimes y)=\bar{f}(x)+\bar{f}(y)$ for $x, y \in T(\mathfrak{g})$. Note that $\bar{f}$ gives the parity of a weight vector of $T(\mathfrak{g})$.

Let $t$ be an element of $T(\mathfrak{g})_{k}$ with weight $a_{1} \epsilon_{1}+\cdots+a_{m} \epsilon_{m}+b_{1} \delta_{1}+\cdots+b_{n} \delta_{n}$. If $t$ is in $\left(T(\mathfrak{g})_{k}\right)^{\mathfrak{g}}$ then the Cartan subalgebra acts by zero and so the weight of $t$ is zero: $a_{i}=b_{j}=0$ for all $i$ and $j$. But from above we know that the parity of $t$ is equal to $\bar{f}(t)=\left(n \sum a_{i}+m \sum b_{j}\right) /(m-n)$ modulo 2 , which is zero if $t$ is in $\left(T(\mathfrak{g})_{k}\right)^{\mathfrak{g}}$. Thus, all the invariant tensors of $T(\mathfrak{g})$ are even. 
From Propositions 2.5.3 and 2.5.5 of [Kac 1977] there exists a unique (up to constant factor) nondegenerate supersymmetric invariant even bilinear form $($,$) on$ $\mathfrak{g}$. Let $b: \mathfrak{g} \rightarrow \mathfrak{g}^{*}$ be the isomorphism given by the assignment $x \mapsto(x, \cdot)$.

We extend this bilinear form to $T(\mathfrak{g})$ by

$$
\left(x_{1} x_{2} \ldots x_{k}, x_{1}^{\prime} x_{2}^{\prime} \ldots x_{l}^{\prime}\right)=\delta_{k l} \prod_{i=1}^{k}(-1)^{\sum_{i<j} \bar{x}_{j} \bar{x}_{i}^{\prime}}\left(x_{i}, x_{i}^{\prime}\right)
$$

where $x_{i}, x_{j}^{\prime} \in \mathfrak{g}$. Since (, ) is nondegenerate on $\mathfrak{g}$, this extension is a nondegenerate bilinear form on $T(\mathfrak{g})$. Moreover, since $(.,$.$) is supersymmetric on \mathfrak{g}$ and $\left(x, x^{\prime}\right)=$ 0 for all $x, x^{\prime} \in \mathfrak{g}$ such that $\bar{x} \neq \bar{x}^{\prime}$, we have that the extension is supersymmetric on $T(\mathfrak{g})$.

For $t \in\left(T(\mathfrak{g})_{N}\right)^{\mathfrak{g}} \simeq \operatorname{Hom}_{\mathfrak{g}}\left(\mathbb{C}, T(\mathfrak{g})_{N}\right)$ we have $t^{*} \in \operatorname{Hom}_{\mathfrak{g}}\left(T\left(\mathfrak{g}^{*}\right)_{N}, \mathbb{C}\right)$, where $*$ is the "supertranspose". Using this notation the bilinear form is given by $\left(t, t^{\prime}\right)=$ $\left\langle t^{*} \circ b^{\otimes N} \circ t^{\prime}\right\rangle$. Here and after, if $g \in \operatorname{End}_{\mathbb{C}}(\mathbb{C})$, we denote by $\langle g\rangle$ the scalar $g(1)$.

Recall the definition of the coevaluation morphism $\operatorname{coev}_{V}$ given in Section 2.

Definition 3.2. For $N \in \mathbb{N}$ define

$$
\mathscr{I} \mathscr{T}_{N}=\left\{f\left(\operatorname{coev}_{V}(1)\right): f \in \operatorname{Hom}_{\mathfrak{g}}\left(V \otimes V^{*}, \mathfrak{g}^{\otimes n}\right) \text { for some } V \in \mathscr{I}\right\}
$$

and $\mathscr{I} \mathscr{T}=\oplus_{N} \mathscr{I} \mathscr{T}_{N}$.

Let $t \in \mathscr{I} \mathscr{T}_{N}$ and $t^{\prime} \in\left(T(\mathfrak{g})_{N}\right)^{\mathfrak{g}}$. We will now show that $\left(t, t^{\prime}\right)$ can be written in terms of the supertrace. We regard $t, t^{\prime}$ as elements of $\operatorname{Hom}_{\mathfrak{g}}\left(\mathbb{C}, \mathfrak{g}^{\otimes N}\right)$. As $t=f\left(\operatorname{coev}_{V}\right)$ for some $f \in \operatorname{Hom}_{\mathfrak{g}}\left(V \otimes V^{*}, \mathfrak{g}^{\otimes N}\right)$ where $V \in \mathscr{I}$, we have $t^{*}=\operatorname{coev}_{V}^{*} \circ f^{*}$ and

$$
\left(t, t^{\prime}\right)=\left\langle\operatorname{coev}_{V}^{*} \circ f^{*} \circ b^{\otimes N} \circ t^{\prime}\right\rangle .
$$

The morphism $f^{*} \circ b^{\otimes N} \circ t^{\prime} \in \operatorname{Hom}_{\mathfrak{g}}\left(\mathbb{C}, V^{*} \otimes V\right) \simeq \operatorname{Hom}_{\mathfrak{g}}\left(\mathbb{C}, V \otimes V^{*}\right)$ can be identified with a $\mathfrak{g}$-linear endomorphism of $V$ which we denote by $\left[f^{*} \circ b^{\otimes N} \circ t^{\prime}\right]$. Thus, we have $\left(t, t^{\prime}\right)=\operatorname{str}_{V}\left(\left[f^{*} \circ b^{\otimes N} \circ t^{\prime}\right]\right)$, which is zero by Proposition 2.2. The above discussion can be summarized in the following lemma.

Lemma 3.3. If $t \in \mathscr{I}^{\mathscr{T}_{N}}$ and $t^{\prime} \in\left(T(\mathfrak{g})_{N}\right)^{\mathfrak{g}}$ then $\left(t, t^{\prime}\right)=\operatorname{str}_{V}\left(\left[f^{*} \circ b^{\otimes N} \circ t^{\prime}\right]\right)$ which is zero.

Proposition 3.4. The sets $\mathscr{G} \mathscr{T}_{N}$ are vector spaces. Moreover, $\mathscr{I} \mathscr{T}=\oplus_{N} \mathscr{\mathscr { T }} \mathscr{T}_{N}$ is a two sided ideal of $T(\mathfrak{g})^{\mathfrak{g}}$ which is in the kernel of the restriction of $(.$, . ) to the space of invariant tensor $T(\mathfrak{g})^{\mathfrak{g}}$.

Proof. We will first show that $\mathscr{I} \mathcal{T}_{N}$ is a vector space. Let $t_{1}, t_{2} \in \mathscr{I} \mathscr{T}_{N}$ and $\lambda \in \mathbb{C}$. Then $t_{i}=f_{i}\left(\operatorname{coev}_{V_{i}}(1)\right)$ for some $f_{i}$ and $V_{i}$. Set $V=V_{1} \oplus V_{2}$. Let $f: V \otimes V^{*} \rightarrow \mathfrak{g}^{\otimes N}$ be the invariant map given by

$$
f\left(\left(v_{1} \oplus v_{2}\right) \otimes\left(\varphi_{1} \oplus \varphi_{2}\right)\right)=f_{1}\left(v_{1} \otimes \varphi_{1}\right)+\lambda f_{2}\left(v_{2} \otimes \varphi_{2}\right) .
$$


Then $f\left(\operatorname{coev}_{V}(1)\right)=t_{1}+\lambda t_{2}$. Thus, $\mathscr{I} \mathscr{T}_{N}$ is a vector space.

Now we will show that $\Phi \mathscr{T}$ is an ideal. Let $t^{\prime} \in\left(\mathfrak{g}^{\otimes M}\right)^{\mathfrak{g}}$ and let $t_{1}$ be as above. Let $g: V_{1} \otimes V_{1}^{*} \rightarrow \mathfrak{g}^{\otimes(M+N)}$ be the invariant map given by

$$
g\left(v_{1} \otimes \varphi_{1}\right)=t^{\prime} \otimes f_{1}\left(v_{1} \otimes \varphi_{1}\right) .
$$

Then $g\left(\operatorname{coev}_{V_{1}}(1)\right)=t^{\prime} \otimes t_{1}$ and so $t^{\prime} \otimes t_{1} \in \mathscr{I}_{M+N}$.

The last statement of the proposition follows from Lemma 3.3.

Next we define a bilinear form on $\mathscr{I} \mathscr{T}$. The following definition is motivated by Lemma 3.3 and justified by Theorem 2 .

Definition 3.5. For $t_{1} \in \mathscr{I} \mathscr{T}_{N}$ and $t_{2} \in \mathscr{G} \mathscr{T}_{M}$ with $t_{i}=f_{i}\left(\operatorname{coev}_{V_{i}}\right)$, define

$$
\left(t_{1}, t_{2}\right)^{\prime}=\delta_{M, N} \operatorname{str}_{V_{1}}^{\prime}\left(\left[f_{1}^{*} \circ b^{\otimes N} \circ t_{2}\right]\right)
$$

We can represent $\left[f_{1}^{*} \circ b^{\otimes N} \circ t_{2}\right]$ by the following picture, where $M=N=3$ for simplicity:

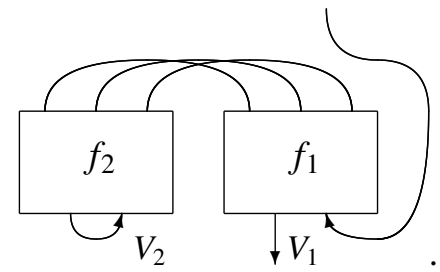

It is tempting to think that the above construction could work for $t_{1} \in \mathscr{I} \mathcal{T}$ and any $t_{2} \in T(\mathfrak{g})$ but this is false because there are examples of $t_{2} \in T(\mathfrak{g})$ for which the above scalar depends not only of $t_{1}$ but also of $f_{1}$.

To simplify notation we will identify $\mathfrak{g}$ and $\mathfrak{g}^{*}$ using the isomorphism $b$ but will no longer write $b$.

Theorem 2. $(., .)^{\prime}$ is a well defined symmetric bilinear form on $\mathscr{I} \mathcal{T}$ satisfying $\left(G\left(t_{1}\right), t_{2}\right)^{\prime}=\left(t_{1}, G^{*}\left(t_{2}\right)\right)^{\prime}$ for any $t_{1} \in \mathscr{I} \mathcal{T}_{M}, t_{2} \in \mathscr{I} \mathcal{T}_{N}, G \in \operatorname{Hom}_{\mathfrak{g}}\left(T(\mathfrak{g})_{M}, T(\mathfrak{g})_{N}\right)$. In particular, the symmetric group $S_{N}$ acts orthogonally on $\mathscr{I}_{\mathcal{T}_{N}}$.

Proof. Let $t_{1}$ and $t_{2}$ be elements of $\mathscr{I} \mathscr{T}_{N}$ with $t_{i}=f_{i}\left(\operatorname{coev}_{V_{i}}\right)$. We need to show that the definition of $\left(t_{1}, t_{2}\right)^{\prime}$ is independent of $f_{1}, f_{2}, V_{1}$, and $V_{2}$.

Using the canonical isomorphism (2), we can make the identifications

$$
\begin{aligned}
\operatorname{Hom}_{\mathfrak{g}}\left(V_{2} \otimes V_{2}^{*}, V_{1} \otimes V_{1}^{*}\right) & \cong \operatorname{Hom}_{\mathfrak{g}}\left(\mathbb{C}, V_{1} \otimes V_{1}^{*} \otimes V_{2}^{*} \otimes V_{2}\right) \\
& \cong \operatorname{Hom}_{\mathfrak{g}}\left(\mathbb{C}, V_{1} \otimes V_{2} \otimes V_{1}^{*} \otimes V_{2}^{*}\right) \cong \operatorname{End}_{\mathfrak{g}}\left(V_{1} \otimes V_{2}\right) .
\end{aligned}
$$

Therefore, below we will consider $f_{1}^{*} \circ f_{2}$ as an element of $\operatorname{End}_{\mathfrak{g}}\left(V_{1} \otimes V_{2}\right)$. Notice that for fixed $t_{1}=f_{1}\left(\operatorname{coev}_{V_{1}}\right)$ the map $\mathscr{I} \mathscr{T}_{N} \rightarrow \mathbb{C}$ given by

$$
t \mapsto \operatorname{str}_{V_{1}}^{\prime}\left(f_{1}^{*} \circ t\right)
$$


is well defined and linear. Then from Theorem 1(d) we have $\operatorname{str}_{V_{1}}^{\prime}\left(f_{1}^{*} \circ t_{2}\right)=$ $\operatorname{str}_{V_{1} \otimes V_{2}}^{\prime}\left(f_{1}^{*} \circ f_{2}\right)=\operatorname{str}_{V_{1} \otimes V_{2}}^{\prime}\left(f_{2}^{*} \circ f_{1}\right)=\operatorname{str}_{V_{2}}^{\prime}\left(f_{2}^{*} \circ t_{1}\right)$, which does not depend on $f_{1}$ or $V_{1}$. Thus, $(., .)^{\prime}$ is a well defined symmetric bilinear form.

For the last statement of the theorem,

$$
\left(G\left(t_{1}\right), t_{2}\right)^{\prime}=\operatorname{str}_{V_{1} \otimes V_{2}}^{\prime}\left(f_{1}^{*} \circ G^{*} \circ f_{2}\right)=\left(t_{1}, G^{*}\left(t_{2}\right)\right)^{\prime} .
$$

\section{Proof of Theorem 1}

The proof of Theorem 1 uses quantized Lie superalgebras and low-dimensional topology. In particular, we have the following general plan: (1) start with the desired statement at the level of $\mathfrak{g}-\mathrm{Mod},(2)$ translate these statements to the quantum level, (3) use properties of invariants of ribbon graphs to prove these statements and (4) take the classical limit to obtain the proof of the original statements. With this in mind we will begin this section by recalling some properties about the DrinfeldJimbo type quantization of $\mathfrak{g}$.

Let $h$ be an indeterminate and set $q=\mathrm{e}^{h / 2}$. We use the notation $q^{z}=\mathrm{e}^{z h / 2}$ for $z \in \mathbb{C}$. Let $U_{h}^{D J}(\mathfrak{g})$ be the Drinfeld-Jimbo type quantization of $\mathfrak{g}$ defined in [Yamane 1994]. The quantization $U_{h}^{D J}(\mathfrak{g})$ is a braided $\mathbb{C} \llbracket h \rrbracket$-Hopf superalgebra given by generators and relations. As we will explain now $U_{h}^{D J}(\mathfrak{g})$ is related to a quasi-Hopf superalgebra.

For each Lie algebra Drinfeld defined a quasi-Hopf quantized universal enveloping algebra:

$$
\left(U(\mathfrak{g}) \llbracket h \rrbracket, \Delta_{0}, \epsilon_{0}, \Phi_{K Z}\right) .
$$

The morphisms $\Delta_{0}$ and $\epsilon_{0}$ are the standard coproduct and counit of $U(\mathfrak{g}) \llbracket h \rrbracket$. The element $\Phi_{K Z}$ is the KZ-associator. Let $A_{\mathfrak{g}}$ be the analogous topologically free quasi-Hopf superalgebra (for more details see [Geer 2006]).

Let $U_{h}^{D J}(\mathfrak{g})-\operatorname{Mod}_{\mathrm{fr}}$ and $A_{\mathfrak{g}}-\operatorname{Mod}_{\mathrm{fr}}$ be the tensor categories of topologically free $U_{h}^{D J}(\mathfrak{g})$-modules and $A_{\mathfrak{g}}$-modules of finite rank, respectively (that is, those of the form $V \llbracket h \rrbracket$, where $V$ is a finite-dimensional $\mathfrak{g}$-module). We say a module $V \llbracket h \rrbracket$ in $U_{h}^{D J}(\mathfrak{g})-\operatorname{Mod}_{\mathrm{fr}}$ is typical if $V$ is a typical $\mathfrak{g}$-module.

In [Geer 2006] the first author proves that there exists a functor $G: A_{\mathfrak{g}}-\operatorname{Mod}_{\mathrm{fr}} \rightarrow$ $U_{h}^{D J}(\mathfrak{g})-\operatorname{Mod}_{\mathrm{fr}}$ which is an equivalence of tensor categories. There is a natural tensor functor $G^{\prime}: \mathfrak{g}-\operatorname{Mod} \rightarrow A_{\mathfrak{g}}-\operatorname{Mod}_{\text {fr }}$ given by $V \mapsto V \llbracket h \rrbracket$ and $f \mapsto G^{\prime}(f)$ where the action of $\mathfrak{g}$ on $V$ extends to an action of $U(\mathfrak{g}) \llbracket h \rrbracket$ on $V \llbracket h \rrbracket$ be linearity and $G^{\prime}(f)\left(\sum v_{i} h^{i}\right)=\sum f\left(v_{i}\right) h^{i}$. We have the commutative diagram of functors

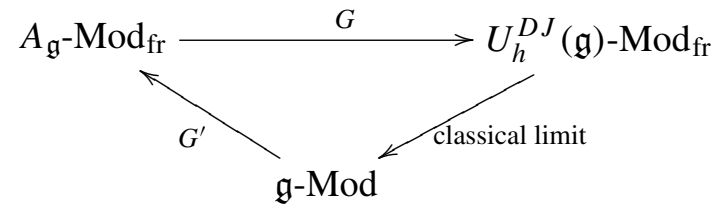


where the down left arrow is the classical limit given by taking the limit as $h$ goes to zero. For any object $V$ and morphism $g$ of $\mathfrak{g}$-Mod let us denote $G \circ G^{\prime}(V)$ and $G \circ G^{\prime}(g)$ by $\widetilde{V}$ and $\widetilde{g}$, respectively. Here the functor $G \circ G^{\prime}$ composed with the classical limit is the identity functor: $V \equiv \widetilde{V} \bmod h$ and $g \equiv \widetilde{g} \bmod h$.

In [Geer and Patureau-Mirand 2006] we defined an invariant of framed colored links. Let us now recall the basic construction and some properties of this invariant. Here we say that a link or more generally a tangle is colored if each of its components are assigned an object of $U_{h}^{D J}(\mathfrak{g})-\operatorname{Mod}_{\mathrm{fr}}$.

Let $F$ be the usual Reshetikhin-Turaev functor from the category of framed colored tangles to the category of $U_{h}^{D J}(\mathfrak{g})-\operatorname{Mod}_{\mathrm{fr}}$. In [Geer and Patureau-Mirand 2006] a function from the set of typical $U_{h}^{D J}(\mathfrak{g})$-module to the ring $\mathbb{C} \llbracket h \rrbracket\left[h^{-1}\right]$ is defined. As remarked in that article, this function can be multiplied by $h^{\left|\Delta_{1}^{+}\right|}$to obtain a function which takes values in $\mathbb{C} \llbracket h \rrbracket$. Let us denote this function by $d_{h}$.

Lemma 4.1.

$$
\mathrm{d}_{\mathrm{h}}(\widetilde{V}(\lambda))=h^{\left|\Delta_{\overline{1}}^{+}\right|} \frac{\prod_{\alpha \in \Delta_{\overline{0}}^{+}} \frac{q^{\langle\lambda+\rho, \alpha\rangle}-q^{-\langle\lambda+\rho, \alpha\rangle}}{q^{\langle\rho, \alpha\rangle}-q^{-\langle\rho, \alpha\rangle}}}{\prod_{\alpha \in \Delta_{\overline{1}}^{+}}\left(q^{\langle\lambda+\rho, \alpha\rangle}-q^{-\langle\lambda+\rho, \alpha\rangle}\right)} .
$$

In particular, $\mathrm{d}(V(\lambda))$ is equal to $\mathrm{d}_{\mathrm{h}}(\widetilde{V}(\lambda)) \bmod h$.

Proof. The proof follows from the formulas for $h^{-\left|\Delta_{1}^{+}\right|} d_{h}$ given in the Appendix of [Geer and Patureau-Mirand 2006] and from the definition of $d$.

Suppose $L$ is a framed colored link such that by cutting some component of $L$ one obtains a framed colored $(1,1)$-tangle $T_{V(\lambda)}$ such that the open string is colored by the deformed typical module $\widetilde{V}(\lambda)$ of highest weight $\lambda$. Then $F\left(T_{V(\lambda)}\right)=$ $x . \operatorname{Id}_{\tilde{V}(\lambda)}$, for some $x$ in $\mathbb{C} \llbracket h \rrbracket$. Set $\left\langle T_{V(\lambda)}\right\rangle=x$. In [Geer and Patureau-Mirand 2006] it is shown that the assignment

$$
L \mapsto \mathrm{d}_{\mathrm{h}}(\tilde{V}(\lambda))\left\langle T_{V(\lambda)}\right\rangle
$$

is a well defined colored framed link invariant denoted by $F^{\prime}$. In particular, $F^{\prime}(L)$ is independent of $\widetilde{V}(\lambda), T_{V(\lambda)}$ and where $L$ is cut.

An even morphism $f: V_{1} \otimes \cdots \otimes V_{n} \rightarrow W_{1} \otimes \cdots \otimes W_{m}$ in the category $U_{h}^{D J}(\mathfrak{g})-\operatorname{Mod}_{\text {fr }}$ can be represented by

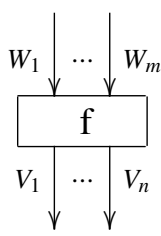


Such a box is called a coupon, which we denote by $C_{V_{1}, \ldots, V_{n}}^{W_{1}, \ldots, W_{m}}(f)$. Here we will say a ribbon graph is a framed tangle with coupons and colors coming from the category $U_{h}^{D J}(\mathfrak{g})-\operatorname{Mod}_{\mathrm{fr}}$. In [Geer et al. 2007] it is shown that the construction of $F^{\prime}$ can be extended to ribbon graphs having at least one component colored by a typical $U_{h}^{D J}(\mathfrak{g})$-module.

The invariant $F^{\prime}$ can also be extended to ribbon graphs having at least one component colored by a deformed module in $\mathscr{I}$ [Geer et al. 2007]. We will now describe this extension in the following situation. Let $C\left(C^{\prime}\right)$ be a $(1,1)$-tangle (resp. $(2,2)$-tangle) ribbon graph such that the input(s) and output(s) are equal. Let $L_{C}$ be the closed ribbon graph obtained from closing the coupon $C$. Let $T_{C^{\prime}}$ be the $(1,1)$-tangle ribbon graph obtained from closing right most component. The ribbon graphs $L_{C}$ and $T_{C^{\prime}}$ can be represented by

$$
L_{C}=\square \quad T_{C^{\prime}}=\stackrel{+9}{C^{\prime}}
$$

These pictures represent respectively the trace and the partial trace of the morphisms in the coupon.

Let $V \in \mathscr{I}$ and let $\alpha: V_{0} \times W \rightarrow V$ and $\beta: V \rightarrow V_{0} \otimes W$ be morphisms in $\mathfrak{g}$-Mod such that $\alpha \circ \beta=\operatorname{Id}_{V}$. Let $f \in \operatorname{End}_{\mathfrak{g}}(V)_{\overline{0}}$ and let $T(f ; \alpha ; \beta)$ be the (1,1)-tangle ribbon graph $T_{C_{\tilde{V}}^{\tilde{V}_{0} \otimes \tilde{W}}(\widetilde{\beta}) \circ C_{\tilde{V}}^{\tilde{V}}(\widetilde{f}) \circ C_{\tilde{V}_{0} \otimes \widetilde{W}}^{\tilde{V}}(\widetilde{\alpha})}$. That is,

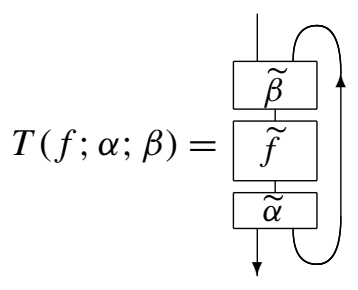

Then we define

$$
F^{\prime}\left(L_{C_{\widetilde{V}}^{\widetilde{V}}(\widetilde{f})}\right)=\mathrm{d}_{\mathrm{h}}\left(\widetilde{V}_{0}\right)\langle T(f ; \alpha ; \beta)\rangle .
$$

In [Geer and Patureau-Mirand 2006; Geer et al. 2007] it is shown that $F^{\prime}$ is well defined. Now we are ready to prove the main theorem of the paper.

Proof of Theorem 1. Let $V_{1}$ be a typical g-module. Then $\mathscr{I}=\mathscr{I}_{V_{0}}=\mathscr{I}_{V_{1}}$. Choose $\alpha_{i}: V_{i} \times W_{i} \rightarrow V$ and $\beta_{i}: V \rightarrow V_{i} \otimes W$ such that $\alpha_{i} \circ \beta_{i}=\operatorname{Id}_{V}$, for $i=0,1$. If $f \in \operatorname{End}_{\mathfrak{g}}(V)_{\overline{1}}$ then $\left\langle f ; \alpha_{0} ; \beta_{0}\right\rangle=\left\langle f ; \alpha_{1} ; \beta_{1}\right\rangle=0$ as $\operatorname{ptr}(\beta \circ f \circ \alpha)=\langle f ; \alpha ; \beta\rangle \operatorname{Id}_{V_{0}}$ and $\beta \circ f \circ \alpha$ is odd. Therefore, we can assume that $f \in \operatorname{End}_{\mathfrak{g}}(V)_{\overline{0}}$ (that is, $f$ is a morphism in the symmetric monoidal category $\left.\mathfrak{g}-\operatorname{Mod}_{\overline{0}}\right)$. We will show that

$$
\mathrm{d}\left(V_{0}\right)\left\langle f ; \alpha_{0} ; \beta_{0}\right\rangle=\mathrm{d}\left(V_{1}\right)\left\langle f ; \alpha_{1} ; \beta_{1}\right\rangle .
$$


By definition of the ribbon category $U_{h}^{D J}(\mathfrak{g})-\operatorname{Mod}_{\text {fr }}$ we have equality between $\left\langle f ; \alpha_{i} ; \beta_{i}\right\rangle$ and $\left\langle T\left(f ; \alpha_{i} ; \beta_{i}\right)\right\rangle \bmod h$, for $i=0,1$. Combining this with Lemma 4.1 we obtain $\mathrm{d}\left(V_{i}\right)\left\langle f ; \alpha_{i} ; \beta_{i}\right\rangle$ is equal to $\mathrm{d}_{\mathrm{h}}\left(\widetilde{V}_{i}\right)\left\langle T\left(f ; \alpha_{i} ; \beta_{i}\right)\right\rangle \bmod h$, for $i=0$, 1. Finally, by [Geer et al. 2007], the extension of $F^{\prime}$ to ribbon graphs is well defined. In particular, we have $\mathrm{d}_{\mathrm{h}}\left(\widetilde{V}_{0}\right)\left\langle T\left(f ; \alpha_{0} ; \beta_{0}\right)\right\rangle=\mathrm{d}_{\mathrm{h}}\left(\widetilde{V}_{1}\right)\left\langle T\left(f ; \alpha_{1} ; \beta_{1}\right)\right\rangle$. Thus, Equation (9) holds and $\operatorname{str}_{V}^{\prime}(f)$ only depends on $f$.

Now we prove the remaining statements of the theorem. The function $\operatorname{str}_{V}^{\prime}$ is linear because $F\left(C_{\widetilde{V}}^{\widetilde{V}}(a \tilde{f}+b \widetilde{g})\right)=a F\left(C C_{\widetilde{V}}^{\widetilde{V}}(\widetilde{f})\right)+b F\left(C C_{\widetilde{V}}^{\widetilde{V}}(\widetilde{g})\right)$ for $f, g \in \operatorname{End}_{\mathfrak{g}}(V)_{\overline{0}}$ and $a, b \in \mathbb{C}$. Part (c) follows from the property that $F^{\prime}\left(L \sqcup L^{\prime}\right)=F^{\prime}(L) F\left(L^{\prime}\right)$ for any two links $L$ and $L^{\prime}$ [Geer et al. 2007]. The proof of (d) follows from the behavior of $F^{\prime}$ with respect to cabling [Geer et al. 2007].

To prove part (b) we need to be careful because coupons must be labeled by even morphisms, but the morphisms in the statement of (b) can be odd. If $V$ is an object of $\mathfrak{g}$-Mod then denote $V^{-}$as the $\mathfrak{g}$-module obtained from $V$ by taking the opposite parity. Then $V$ and $V^{-}$are isomorphic by an odd isomorphism $\sigma_{V}: V \rightarrow V^{-}$, which changes the parity.

Lemma 4.2. Let $\gamma \in \operatorname{End}_{U_{h}^{D J}(\mathfrak{g})}(\widetilde{W} \otimes \widetilde{V})_{\overline{0}}$ and set $\eta=\left(\operatorname{Id} \otimes \widetilde{\sigma}_{V}\right) \gamma\left(\operatorname{Id} \otimes \widetilde{\sigma}_{V}\right)$. Then

$$
F\left(T_{C_{\tilde{W} \otimes \tilde{V}}^{\tilde{W} \otimes \tilde{V}}(\gamma)}\right)=-F\left(T_{C_{\widetilde{W} \otimes \tilde{V}^{-}}^{\tilde{W} \otimes \tilde{V}^{-}}(\eta)}\right) .
$$

Proof. Let $\left\{w_{i}\right\}_{i=1}^{q}$ and $\left\{v_{j}\right\}_{j=1}^{p}$ be bases of the g-modules $V$ and $W$, respectively. Then $\left\{v_{j}\right\}_{j=1}^{p},\left\{\sigma_{V}\left(v_{j}\right)\right\}_{j=1}^{p}$ and $\left\{w_{i}\right\}_{i=1}^{q}$ are bases for the $U_{h}^{D J}(\mathfrak{g})$-modules $\widetilde{V}, \widetilde{V}^{-}$ and $\widetilde{W}$, respectively.

Let $\gamma_{i j}^{k l}$ be the elements of $\mathbb{C} \llbracket h \rrbracket$ defined by

$$
\gamma\left(w_{i} \otimes v_{j}\right)=\sum_{k=1}^{q} \sum_{l=1}^{p} \gamma_{i j}^{k l} w_{k} \otimes v_{l} .
$$

A direct calculation shows that

$$
\begin{aligned}
F\left(T_{C_{\tilde{W} \otimes \tilde{V}}^{\tilde{W} \otimes \tilde{V}}(\gamma)}\right)\left(w_{i}\right) & =\sum_{k=1}^{q} \sum_{l=1}^{p}(-1)^{\bar{v}_{j}} \gamma_{i j}^{k j} w_{k}, \\
F\left(T_{C_{\tilde{W} \otimes \tilde{V}^{-}}^{\tilde{W} \otimes \tilde{V}^{-}}(\eta)}\right)\left(w_{i}\right) & =\sum_{k=1}^{q} \sum_{l, j=1}^{p}(-1)^{\bar{w}_{i}+\bar{w}_{k}}(-1)^{\left(\overline{1}+\bar{v}_{l}\right)\left(\overline{1}^{+}+\bar{v}_{j}\right)} \delta_{l j} \gamma_{i j}^{k l} w_{k},
\end{aligned}
$$

where $\delta_{l j}(-1)^{\left(\overline{1}+\bar{v}_{l}\right)\left(\overline{1}+\bar{v}_{j}\right)}=(-1)^{\overline{1}^{1}+\bar{v}_{j}}$ and $\bar{w}_{i}=\bar{w}_{k}$ since $\eta$ is an even morphism. Therefore, the right sides of (10) and (11) are the negative of each other and the lemma follows. 
Lemma 4.3. For $V \in \mathscr{I}$ and $f \in \operatorname{End}_{\mathfrak{g}}(V)_{\overline{0}}$ we have

$$
F^{\prime}\left(L_{C_{\widetilde{V}}^{\widetilde{V}}(\widetilde{f})}\right)=-F^{\prime}\left(L_{C_{\widetilde{V}^{-}}^{\tilde{V}^{-}}(\widetilde{\sigma} \circ \tilde{f} \circ \widetilde{\sigma})}\right) .
$$

Proof. Let $\alpha \in \operatorname{End}_{\mathfrak{g}}\left(V_{0} \otimes W, V\right)_{\overline{0}}$ and $\beta \in \operatorname{End}_{\mathfrak{g}}\left(V, V_{0} \otimes W\right)_{\overline{0}}$ such that $\operatorname{Id}_{V}=\alpha \circ \beta$. Then for $\alpha^{-}=\left(\operatorname{Id}_{V_{0}} \otimes \sigma_{W}\right) \circ \alpha \circ \sigma_{V} \in \operatorname{End}_{\mathfrak{g}}\left(V_{0} \otimes W^{-}, V^{-}\right)_{\overline{0}}$ and $\beta^{-}=\sigma_{V} \circ \beta \circ$ $\left(\operatorname{Id}_{V_{0}} \otimes \sigma_{W}\right) \in \operatorname{End}_{\mathfrak{g}}\left(V^{-}, V_{0} \otimes W^{-}\right)_{\overline{0}}$, we have $\operatorname{Id}_{V^{-}}=\alpha^{-} \circ \beta^{-}$. Now, we also denote $\tilde{f}^{-}=\widetilde{\sigma}_{V} \circ \tilde{f}_{0} \widetilde{\sigma}_{V}^{-1} \in \operatorname{End}_{\mathfrak{g}}\left(V^{-}\right)_{\overline{0}}$ and it is convenient to give a pictorial proof:

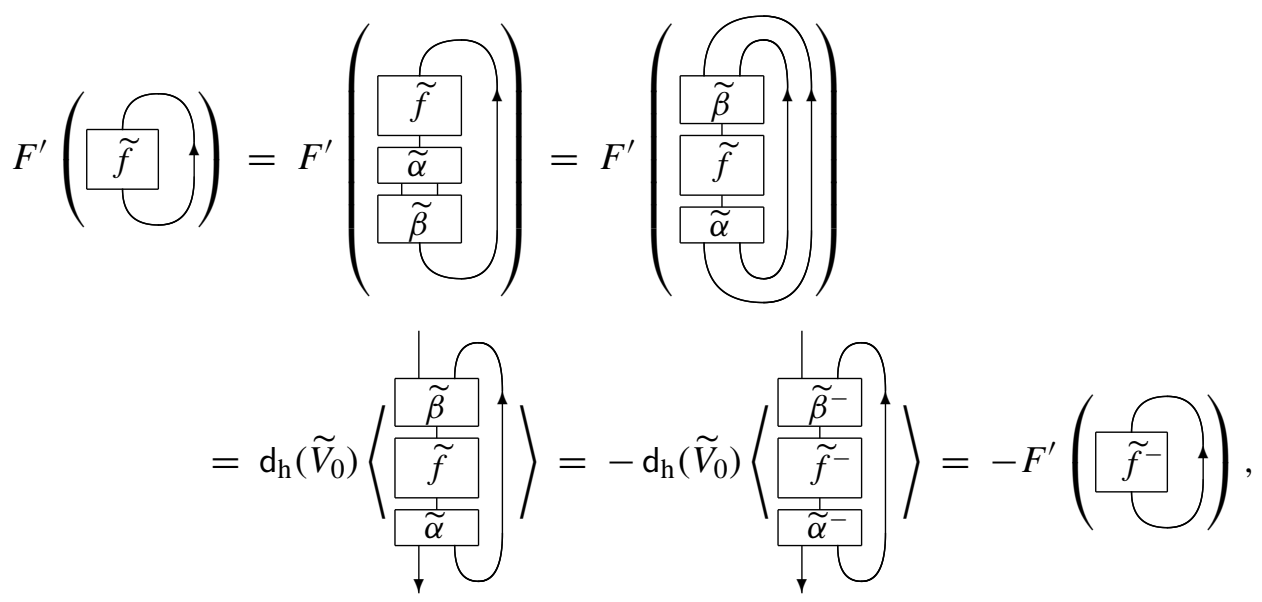

where the fourth equality comes from Lemma 4.2.

Now we are ready to prove part (b). Let $f: V \rightarrow V^{\prime}$ and $g: V^{\prime} \rightarrow V$ be morphisms of $\mathfrak{g}$-Mod such that $f \circ g$ is even. If $f$ and $g$ are both even then part (b) follows from the fact that the closure of $C_{\widetilde{V}}^{\widetilde{V}^{\prime}}(\widetilde{f}) \circ C_{\widetilde{V}^{\prime}}(\widetilde{g})$ is isotopic to closure of $C_{\widetilde{V}^{\prime}}^{\widetilde{V}}(\widetilde{g}) \circ C_{\widetilde{V}}^{\widetilde{V}^{\prime}}(\widetilde{f})$. If $f$ and $g$ are both odd then (b) follows from the following lemma.

Lemma 4.4. If $f$ and $g$ are both odd then

$$
F^{\prime}\left(L_{C_{\tilde{V}}^{\widetilde{V}}(\tilde{f} \circ \widetilde{g})}\right)=-F^{\prime}\left(L_{C_{\widetilde{V}^{\prime}}^{\widetilde{V}^{\prime}}(\widetilde{g} \circ \tilde{f})}\right) .
$$

Proof. From Lemma 4.3 we have

$$
F^{\prime}\left(L_{C_{\tilde{V}}^{\widetilde{V}}(f \circ g)}\right)=-F^{\prime}\left(L_{C_{\tilde{V}^{-}}^{\tilde{V}^{-}}(\widetilde{\sigma} \circ \tilde{f} \circ \tilde{g} \circ \widetilde{\sigma})}\right) .
$$

Now since $\tilde{\sigma} \circ \tilde{f}$ and $\tilde{g} \circ \widetilde{\sigma}$ are even, the right side of Equation (12) is equal to

$$
\begin{aligned}
-F^{\prime}\left(L_{C_{\tilde{V}^{-}}(\widetilde{\sigma} \circ \widetilde{f}) \circ C_{\tilde{V}^{-}}^{\tilde{V}}(\widetilde{g} \circ \widetilde{\sigma})}\right) & =-F^{\prime}\left(L_{C_{\tilde{V}^{-}}(\tilde{g} \circ \widetilde{\sigma}) \circ C_{\tilde{V}^{-}}(\widetilde{\sigma} \circ \widetilde{f})}\right) \\
& =-F^{\prime}\left(L_{C_{\widetilde{V}}^{\widetilde{V}}(\widetilde{g} \circ \widetilde{\sigma} \widetilde{\sigma} \circ \widetilde{f})}\right)=-F^{\prime}\left(L_{C_{\widetilde{V}}^{\widetilde{V}}(\widetilde{g} \circ \widetilde{f})}\right) .
\end{aligned}
$$

Thus we have proved the lemma. 
This finishes the proof of part (b) and the theorem.

Acknowledgments. This work was done while Geer visited LMAM, Université de Bretagne-Sud, which he thanks for the invitations. Both authors thank Michel Duflo for his comments on the first version of this manuscript.

\title{
References
}

[Bar-Natan et al. 2003] D. Bar-Natan, T. T. Q. Le, and D. P. Thurston, "Two applications of elementary knot theory to Lie algebras and Vassiliev invariants", Geom. Topol. 7 (2003), 1-31. MR 2004f:57017 Zbl 1032.57008

[Geer 2006] N. Geer, "Etingof-Kazhdan quantization of Lie superbialgebras", Adv. Math. 207:1 (2006), 1-38. MR 2007g:17019 Zbl 05078165

[Geer and Patureau-Mirand 2006] N. Geer and B. Patureau-Mirand, "Multivariable link invariants arising from Lie superalgebras of type I", preprint, 2006. arXiv math.GT/0609034

[Geer et al. 2007] N. Geer, B. Patureau-Mirand, and V. Turaev, "Modified quantum dimensions and re-normalized link invariants", preprint, 2007. arXiv math.QA/0711.4229

[Kac 1977] V. G. Kac, "Lie superalgebras", Advances in Math. 26:1 (1977), 8-96. MR 58 \#5803 Zbl 0366.17012

[Kac 1978] V. Kac, "Representations of classical Lie superalgebras", pp. 597-626 in Differential geometrical methods in mathematical physics, II (Bonn, 1977), edited by K. Bleuler et al., Lecture Notes in Math. 676, Springer, Berlin, 1978. MR 80f:17006 Zbl 0388.17002

[Kashiwara 1990] M. Kashiwara, "Crystalizing the $q$-analogue of universal enveloping algebras", Comm. Math. Phys. 133:2 (1990), 249-260. MR 92b:17018 Zbl 0724.17009

[Turaev 1994] V. G. Turaev, Quantum invariants of knots and 3-manifolds, Studies in Mathematics 18, de Gruyter, Berlin, 1994. MR 95k:57014 Zbl 0812.57003

[Yamane 1994] H. Yamane, "Quantized enveloping algebras associated with simple Lie superalgebras and their universal $R$-matrices”, Publ. Res. Inst. Math. Sci. 30:1 (1994), 15-87. MR 95d:17017 Zbl 0821.17005

Received December 10, 2007. Revised June 24, 2008.

\author{
NATHAN GEER \\ SCHOOL OF MATHEMATICS \\ GeORgIA Institute of TeCHNOLOGY \\ ATLANTA, GA 30332-0160 \\ UNITED STATES \\ geer@math.gatech.edu \\ BERTRAND PATUREAU-Mirand \\ LMAM, UNIVERSITÉ DE BRETAGNE-SUD \\ B.P. 573 \\ F-56017 VANNES \\ FRANCE \\ bertrand.patureau@univ-ubs.fr
}

